\title{
Inhibitory effect of troglitazone on diabetic neuropathy in streptozotocin-induced diabetic rats
}

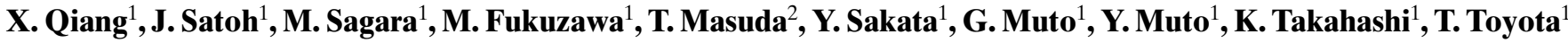 \\ ${ }^{1}$ Third Department of Internal Medicine, Tohoku University School of Medicine, Aoba-ku, Sendai, Japan \\ ${ }^{2}$ Department of Medical Technology, College of Medical Science, Tohoku University, Aoba-ku, Sendai, Japan
}

\begin{abstract}
Summary Free-radical scavengers and inhibitors of tumour necrosis factor- $\alpha(\mathrm{TNF}-\alpha)$ such as $\mathrm{N}$-acetylcysteine and pentoxifylline have been shown to inhibit the development of peripheral neuropathy in streptozotocin(STZ)-induced diabetic rats. In this study we examined the effect of troglitazone, an anti-diabetic thiazolidinedione, on diabetic neuropathy, since it also is a free-radical scavenger and a TNF- $\alpha$ inhibitor. Rats were fed powder chow mixed with troglitazone at $0.5 \%$ and $0.125 \%$ ad libitum. Although blood glucose concentrations were remarkably higher and body weight lower in diabetic than in nondiabetic rats, troglitazone had no effect on these throughout the 24-week experiment. Serum lipoperoxide concentrations, tibial nerve lipoperoxide content and serum TNF- $\alpha$ activity induced by lipopolysaccharide was increased in diabetic rats, but inhibited in troglitazonetreated rats. Motor nerve conduction velocity
\end{abstract}

(MNCV) of the tibial nerve slowed in diabetic rats, compared with that in nondiabetic rats. On the other hand, the slowed MNCV was $(p<0.05-0.01)$ inhibited after weeks 12 and 16 of the experiment in diabetic rats treated with high and low doses of troglitazone, respectively. Morphometric analysis showed that troglitazone suppressed the decrease of the myelinated fibre area $(p<0.05)$, axon/myelin ratio $(p<0.01)$ and fascicular area $(p<0.05)$ and suppressed the increase of myelinated fibre density $(p<0.001)$ in diabetic rats. These results indicate that troglitazone has a beneficial effect on peripheral neuropathy in STZ-induced diabetic rats irrespective of blood glucose concentrations. [Diabetologia (1998) 41: 1321-1326]

Keywords Troglitazone, diabetic neuropathy, free radical scavenger, tumour necrosis factor- $\alpha$ (TNF- $\alpha)$, motor nerve conduction velocity (MNCV).
Diabetic neuropathy could be caused by nerve and vascular dysfunctions due to the increased activity of the polyol pathway [1] and advanced glycation end products (AGEs) produced under long-term hyperglycaemia [2]. These end products stimulate free radical and cytokine production which could also have a

Received: 16 March 1998 and in revised form: 8 June 1998

Corresponding author: Dr. J. Satoh, Third Department of Internal Medicine, Tohoku University School of Medicine, 1-1 Seiryo-machi, Aoba-ku, Sendai 980-8574, Japan

Abbreviations: AGEs, Advanced glycation end products; TNF- $\alpha$, tumour necrosis factor- $\alpha$; STZ, streptozotocin; PPAR- $\gamma$, peroxisome-proliferated activator receptor- $\gamma$; MNCV, motor nerve conduction velocity; LPS, lipopolysaccharide role in the pathogenesis of diabetic neuropathy [2]. We have reported previously that the production of tumour necrosis factor- $\alpha$ (TNF- $\alpha$ ) was enhanced under long-term hyperglycaemia in diabetic animal models [3, 4] and humans [5], and that $\mathrm{N}$-acetylcysteine [6] and pentoxifylline [7], both known as free radical scavengers and inhibitors of TNF- $\alpha$ production, ameliorated the diabetic neuropathy in streptozotocin (STZ)-induced diabetic rats. These results imply that free radical scavengers or TNF- $\alpha$ inhibitors or both can suppress the development of diabetic neuropathy.

Troglitazone, a new oral anti-diabetic thiazolidinedione, binds to a nuclear receptor, peroxisome-proliferated activator receptor- $\gamma$ (PPAR- $\gamma$ ) [8], and reduces insulin resistance by improving insulin signals in insulin-resistant animals [9] and patients [10]. Recent 
studies have shown troglitazone to be a free radical scavenger $[11,12,13]$ and a TNF- $\alpha$ inhibitor $[14,15]$. This indicates that troglitazone can inhibit diabetic neuropathy as do $\mathrm{N}$-acetylcysteine [6] and pentoxifylline [7]. In this study, we investigated whether troglitazone has an inhibitory effect on diabetic neuropathy by using STZ-induced diabetic rats.

\section{Materials and methods}

Animals and induction of diabetes. Male Wistar/Slc rats aged 12 weeks, with a mean body weight of $270 \mathrm{~g}$ were purchased from Clea Japan (Tokyo, Japan). "Principles of Laboratory Animal Care" (NIH publication no. 85-23, revised 1985) were followed in the experiment. Diabetes was induced by i.v. injection of STZ (30 mg/kg body weight) in the tail vein and the rats were defined as diabetic when blood glucose concentration exceeded $16.67 \mathrm{~mol} / \mathrm{l} 24 \mathrm{~h}$ after the STZ injection.

Treatment with troglitazone. Low (L) $(0.125 \%)$ and high $(\mathrm{H})$ $(0.5 \%)$ doses of troglitazone (produced and kindly provided by Sankyo Co., Tokyo, Japan) was mixed with powder chow and orally given ad libitum during the 24-week experiment. Doses of the drug were determined based on the previous reports of animal experiments [9]. Rats were given doses of troglitazone of approximately 10-50 times as much as clinical doses for humans, i.e. $123-529 \mathrm{mg} \cdot \mathrm{kg}^{-1} \cdot \mathrm{day}^{-1}$ for rats compared with $400 \mathrm{mg} \cdot$ body weight ${ }^{-1} \cdot$ day $^{-1}$ for humans [10]. Rats were divided into 5 groups, each consisting of 10 rats: nondiabetic, nondiabetic/troglitazone-high, diabetic, diabetic/ troglitazone-high, diabetic/troglitazone-low. The actual dose of troglitazone intake was calculated from the amount of chow eaten daily.

Measurement of blood glucose, body weight and motor nerve conduction velocity (MNCV). The body weight, blood glucose and MNCV were measured at $0,4,12,16$ and 20 weeks of the 24-week-experiment. The blood glucose concentrations were measured by a glucometer (Glutest-E, Kyoto Daiichi Kagaku, Kyoto, Japan) using a glucose oxidase method. Measurement of MNCV was done in the left tibial nerve by a standard method reported previously [16].

Assay of serum and nerve lipoperoxide. Lipoperoxide was assayed in serum obtained at the end ( 24 weeks) of the experiment by the thiobarbiturate reaction using a commercial kit (Lipoperoxide-Test Wako, Wako Co., Osaka, Japan). Briefly, malondialdehyde derived from serum lipoperoxide under the acidic condition was reacted with thiobarbiturate and malondialdehyde-thiobarbiturate complex extracted with n-butanol was detected by absorption of $553 \mathrm{~nm}$ of fluorescence [17].

For assay of nerve lipoperoxide, $10-20 \mathrm{mg}$ of the frozen right tibial nerve was homogenized in $1 \mathrm{ml}$ of phosphate-buffered saline by a homogenizer (Polytron, Kinematica, Kriens, Luzern, Switzerland). Homogenate and supernatant $(0.05 \mathrm{ml})$ was processed after centrifugation and measured for lipoperoxide concentration as for the serum described above. Protein concentration in homogenate and supernatant was measured by a Bio-Rad Protein Assay kit (Bio-Rad Laboratories, New York, N. Y., USA). Nerve lipoperoxide content is expressed as $\mathrm{nmol} / \mathrm{mg}$ protein. Since both homogenate and supernatant gave similar data on lipoperoxide, those from supernatant were used as nerve lipoperoxide.
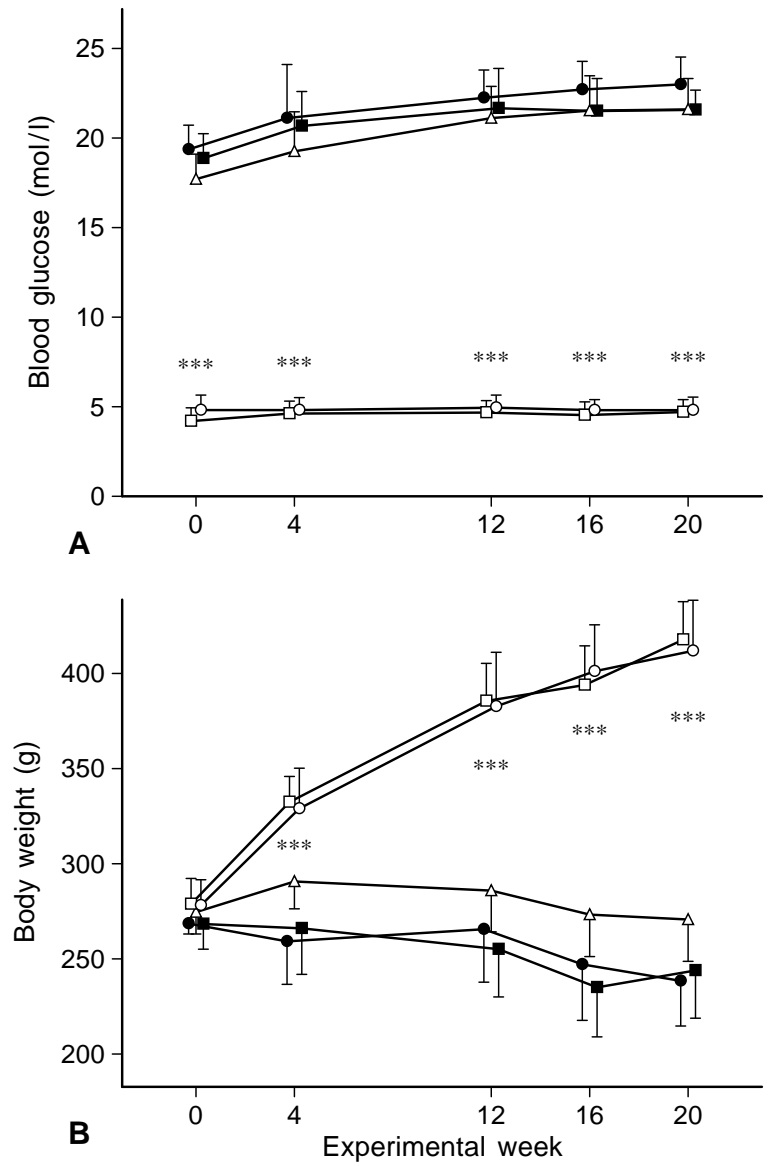

Fig.1A, B. Effect of troglitazone on (A) blood glucose concentrations and (B) body weights. $\square$, nondiabetic; $\bigcirc$, nondiabetic/troglitazone-high; $\mathbf{\square}$, diabetic; $\triangle$, diabetic/troglitazonelow; diabetic/troglitazone-high. Each group consisted of 10 rats. $* * * p<0.001$; nondiabetic rats vs diabetic rats

Assay of serum insulin. Serum obtained at the end (24 weeks) of the experiment was assayed for rat insulin by using a commercial kit (Rat insulin $\left[{ }^{125} \mathrm{I}\right]$ assay system with magnetic separation, Amersham Life Science, Buckinghamshire, England).

Assay of TNF- $\alpha$ activity. At 22 weeks of the 24-week experiment, each rat was injected with lipopolysaccharide $(0.4 \mathrm{mg} /$ kg) (LPS, E. coli 055: B5 Difco Laboratories, Detroit, Mich., USA) in the tail vein, $90 \mathrm{~min}$ later blood was sampled and the serum was isolated and measured for TNF- $\alpha$ activity by using LM cell, a subline of the TNF-sensitive mouse fibroblast (L929), as reported previously [18].

Morphological analysis of nerve. At the end of the 24-week-experiment, all rats were killed under anaesthesia with ether and the left tibial nerve was dissected and immediately fixed with $2.5 \%$ glutaraldehyde fixative. These specimens were embedded in Epon, sliced into semi-thin cross sections and stained with toluidine blue for light microscopic examination and morphometric analysis. The stained nerve sections were photographed by a digital camera (Olympus BH-2, Olympus Optical Co., Tokyo, Japan) equipped with the TV camera (Nikon DS505, Nikon, Tokyo, Japan). The fascicular area using the perineurium as the outer border, the myelinated fibre density and myelinated fibre area were measured and analysed by a 


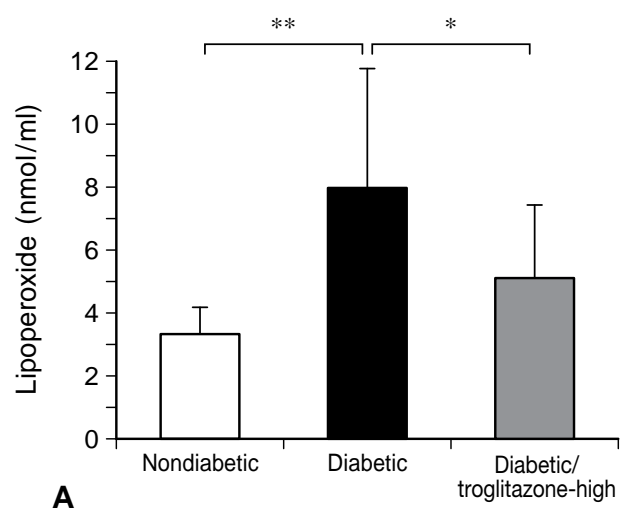

Fig. 2 A, B. Inhibition with troglitazone treatment of $(\mathbf{A})$ increased serum lipoperoxide concentrations and $(\mathbf{B})$ increased nerve lipoperoxide in diabetic rats. The sera and tibial nerves were obtained at the end ( 24 weeks) of experiment. Each group consisted of 10 rats. $* p<0.05, * * p<0.01$

computer-assisted image analysis system (NIH Image, Agfa Arcusscanner).

Statistical analysis. All data are expressed as means \pm SD. Statistical significance of differences was calculated by analysis of variance (ANOVA) and the Bonferroni correction for independent samples. $P$-value of 0.05 or less was considered as significant.

\section{Results}

Effect of troglitazone treatment on blood glucose concentrations and body weight. The blood glucose concentrations of diabetic rats were more than $16.67 \mathrm{~mol} / \mathrm{l} 24 \mathrm{~h}$ after the STZ injection. Hyperglycaemia remained stable throughout the entire experiment (Fig.1). There was no difference in blood glucose concentrations between nontreated rats and troglitazone-treated rats of diabetic and nondiabetic groups, i.e., treatment with troglitazone had no effect on blood glucose concentrations in this experiment.

Initially, all five groups had similar body weights. Body weight increased with ageing in nondiabetic and nondiabetic/troglitazone-high rats (Fig.1). On the other hand, the diabetic, diabetic/troglitazonehigh, and diabetic/troglitazone-low groups lost body weight and were lighter than nondiabetic rats $(p<0.001)$. Treatment with troglitazone had no effect on body weight in either diabetic or nondiabetic rats (Fig.1).

Inhibition with troglitazone treatment of increased serum lipoperoxide concentrations and nerve lipoperoxide content in diabetic rats. Serum lipoperoxide concentrations and tibial nerve lipoperoxide content

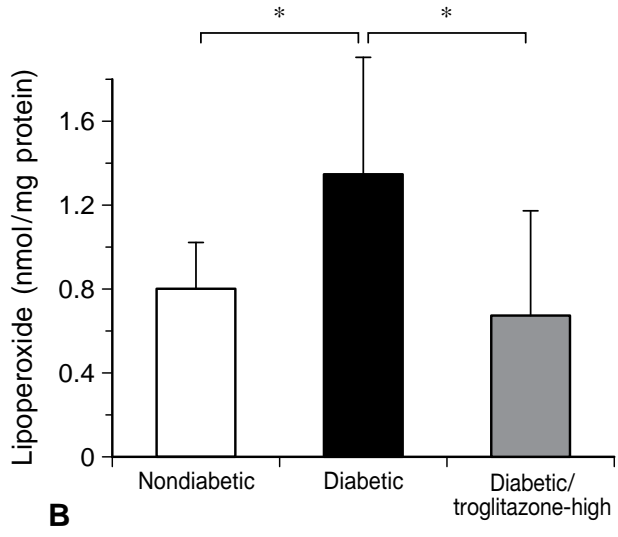

were increased $(p<0.01)$ in diabetic rats compared with nondiabetic rats. The increased lipoperoxide of both serum and nerve was inhibited $(p<0.05)$ in diabetic/troglitazone-high rats (Fig. 2).

Inhibition with troglitazone treatment of enhanced lipopolysaccharide (LPS)-induced serum TNF- $\alpha$ activity. Serum TNF- $\alpha$ activity induced by LPS was greatly enhanced in diabetic rats compared with nondiabetic rats. On the other hand, the enhancement of TNF- $\alpha$ activity in diabetic rats was inhibited, but not so strongly, in diabetic/troglitazone-high $(p<0.01)$ and diabetic/troglitazone-low rats $(p<0.01)$. Troglitazone treatment did not significantly inhibit the TNF- $\alpha$ activity in nondiabetic/troglitazone-high rats (Fig. 3).

Effects of troglitazone treatment on $M N C V$. The MNCV slightly increased with ageing in nondiabetic rats, but decreased in diabetic rats after 4 weeks (Fig.4). The slowing of MNCV in diabetic rats was, however, inhibited $(p<0.05-0.01)$ after week 12 and 16 of the experiment in diabetic/troglitazone-high and diabetic/troglitazone-low rats, respectively. Troglitazone treatment had no effect on MNCV in nondiabetic/troglitazone-high rats.

Effects of troglitazone treatment on nerve morphology. At the end of the 24-week-experiment, the left tibial nerve was morphologically analysed. The mean myelinated fibre area, axon:myelin ratio and fascicular area were diminished in diabetic rats compared with nondiabetic rats and these diminutions were inhibited in diabetic/troglitazone-high rats. Inversely, the mean fibre density was increased in diabetic rats compared with nondiabetic rats but the increase was inhibited in diabetic/troglitazone-high rats (Fig.5).

Effects of troglitazone treatment on serum insulin and blood glucose concentrations at the end of experiment. Table 1 shows the serum insulin and blood glucose levels in the nonfasting condition in the rats randomly chosen at the end of the 24-week-experiment. Nonfasting serum insulin was lower and blood glucose concentrations higher in diabetic rats than in nondiabetic 


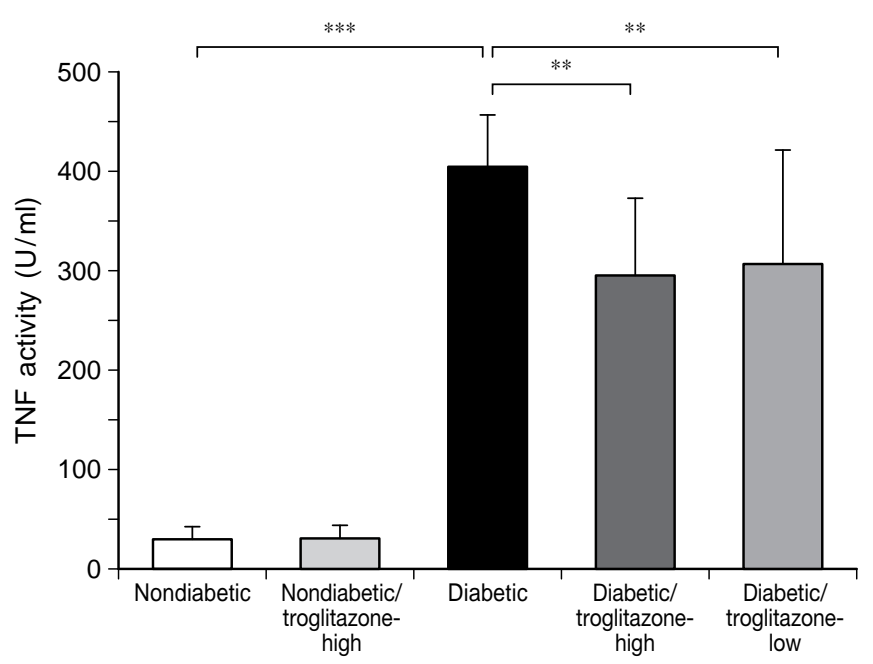

Fig.3. Inhibition with troglitazone treatment of enhanced TNF- $\alpha$ production in diabetic rats. The sera were obtained at week 22 of the 24-week-experiment. Each group consisted of 10 rats. $* * p<0.01, * * * p<0.001$

rats. Troglitazone treatment had no effect on insulin and glucose concentrations.

\section{Discussion}

In this study, treatment with troglitazone inhibited the increased serum lipoperoxide concentrations and nerve lipoperoxide content, the enhanced production of TNF- $\alpha$ and the progression of diabetic neuropathy as do $\mathrm{N}$-acetylcysteine [6] and pentoxifylline [7].

Rats were given troglitazone mixed with chow for a long term. The doses of troglitazone (123.1$529.4 \mathrm{mg} \cdot \mathrm{kg}^{-1} \cdot$ day $^{-1}$ ) were higher than clinical doses given to humans [10] but equivalent to a rodent experiment reported previously [9], in which troglitazone effectively lowered blood glucose concentrations in insulin resistant mice and rats. Troglitazone treatment, however, had no effect on nonfasting serum insulin and blood glucose concentrations in both nondiabetic and diabetic rats. The reason for this could be that the STZ-induced diabetic rats were not insulin-resistant but insulin-deficient. Simi-

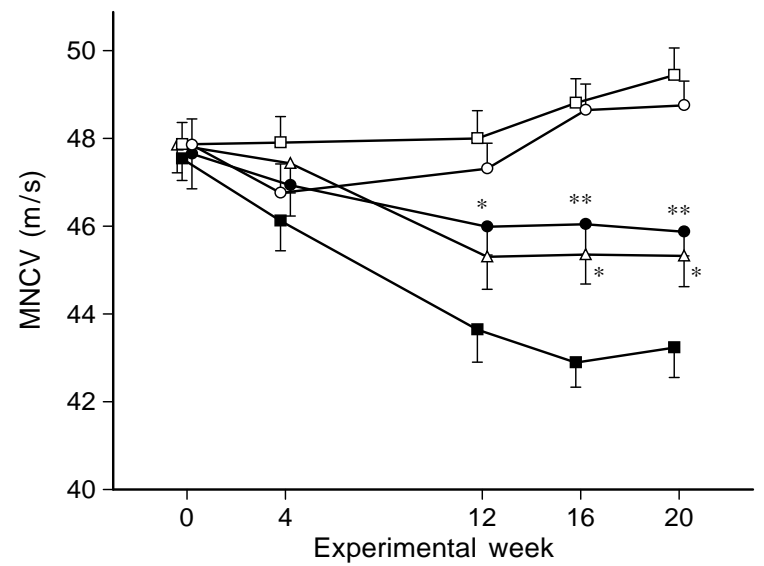

Fig. 4. Effects of troglitazone treatment on MNCV. $\square$, nondiabetic; $\bigcirc$, nondiabetic/troglitazone-high; $\mathbf{\square}$, diabetic; $\triangle$, diabetic/troglitazone-low; $\mathbf{O}$, diabetic/troglitazone-high. Each group consisted of 10 rats. $* p<0.05, * * p<0.01$ vs diabetic rats. $p<0.01$ (4-week) and $p<0.001$ (12-, 16- and 20-week) between nondiabetic rats and diabetic rats

larly, troglitazone did not affect the body weight which increased in nondiabetic rats and decreased in diabetic rats. The dose of troglitazone consumed by diabetic/troglitazone-high rats was twice as much as by nondiabetic/troglitazone-high rats, probably due to polyphagia of the diabetic rats.

The slowed MNCV in diabetic rats was suppressed by troglitazone treatment, although the treatment did not improve serum insulin and blood glucose concentrations. The beneficial effect of troglitazone on diabetic neuropathy was also confirmed by morphological analysis at the end of the experiment. Nerve fibre area, axon:myelin ratio and fascicular area decreased and nerve fibre density increased in diabetic rats compared with nondiabetic rats as reported previously $[16,19]$. These abnormalities were suppressed in the troglitazone-treated rats.

The exact mechanism(s) of action by which troglitazone inhibited the neuropathy is not known. Recent studies have shown that troglitazone is a potent free radical scavenger $[11,12,13]$, and free radical scavengers do prevent peripheral neuropathy in diabetic rats $[20,21]$. The action of troglitazone as a free radi-

Table 1. Non-fasting serum insulin and blood glucose concentrations of nondiabetic and diabetic rats with or without treatment with troglitazone

\begin{tabular}{|c|c|c|c|c|c|}
\hline \multirow[t]{2}{*}{ Rats } & \multirow[t]{2}{*}{$n$} & \multicolumn{2}{|l|}{ Troglitazone } & \multirow{2}{*}{$\begin{array}{l}\text { Serum insulin } \\
(\mathrm{ng} / \mathrm{ml})\end{array}$} & \multirow{2}{*}{$\begin{array}{l}\text { Blood glucose } \\
(\mathrm{mol} / \mathrm{l})\end{array}$} \\
\hline & & $\begin{array}{l}\text { Content in power chow } \\
\%\end{array}$ & $\begin{array}{l}\text { Actual dose given } \\
\left(\mathrm{mg} \cdot \mathrm{kg}^{-1} \cdot \text { day }^{-1}\right)\end{array}$ & & \\
\hline nondiabetic & 6 & 0 & 0 & $2.38 \pm 1.13$ & $4.48 \pm 0.52$ \\
\hline nondiabetic/troglitazone-high & 7 & 0.5 & $263.4 \pm 13.9$ & $2.08 \pm 0.58$ & $4.61 \pm 0.25$ \\
\hline diabetic & 9 & 0 & 0 & $0.71 \pm 0.16^{\mathrm{a}}$ & $18.58 \pm 1.87^{\mathrm{b}}$ \\
\hline diabetic/troglitazone-low & 5 & 0.125 & $123.1 \pm 7.6$ & $0.96 \pm 0.31^{\mathrm{a}}$ & $17.58 \pm 1.34^{\mathrm{b}}$ \\
\hline diabetic/troglitazone-high & 7 & 0.5 & $529.4 \pm 36.9$ & $0.78 \pm 0.25^{\mathrm{a}}$ & $18.88 \pm 1.43^{\mathrm{b}}$ \\
\hline
\end{tabular}

${ }^{\mathrm{a}} p<0.01,{ }^{\mathrm{b}} p<0.001$ vs nondiabetic 

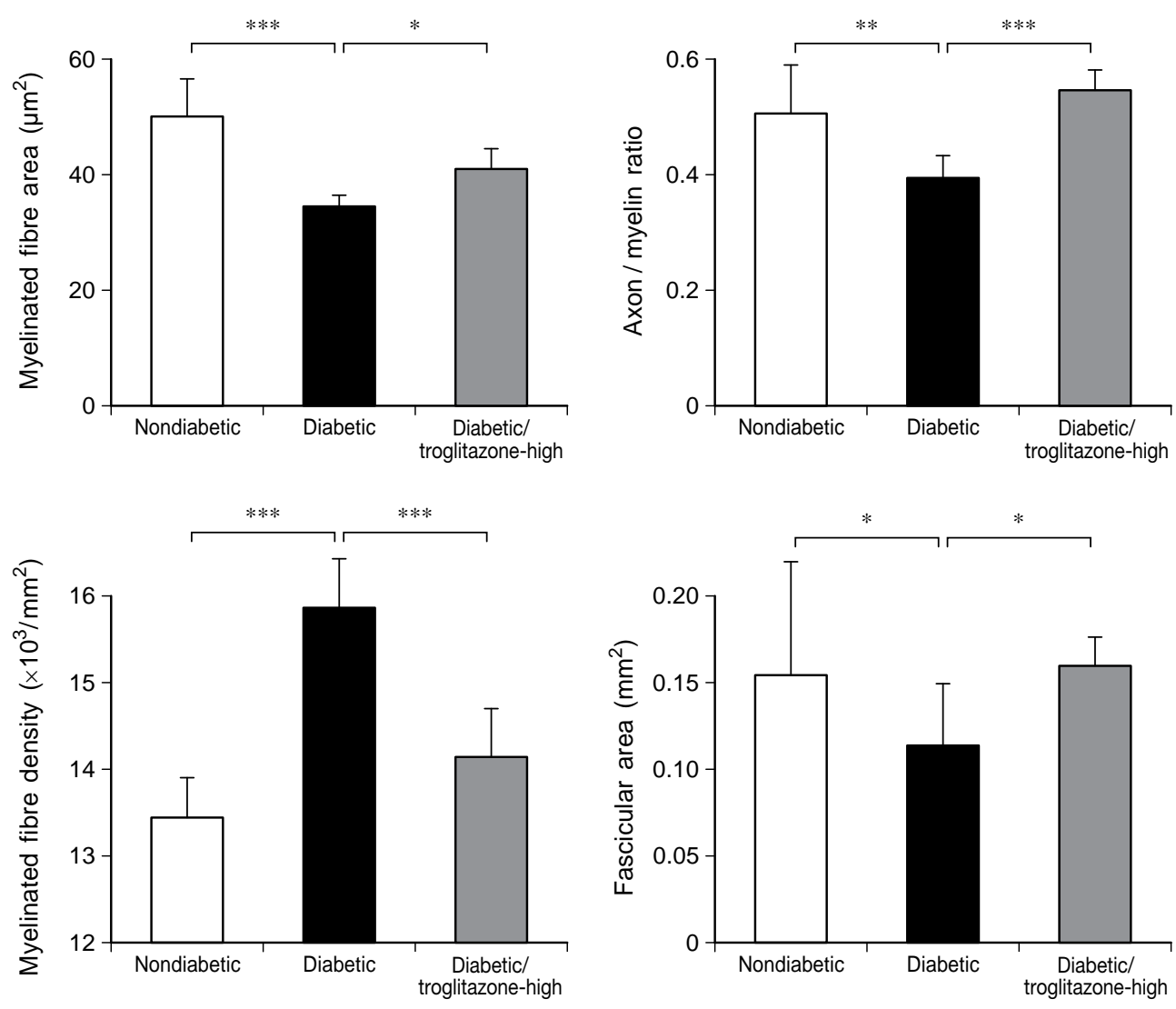

Fig.5. Effect of troglitazone treatment on nerve fibre morphology. The nerve tissues were obtained at the end ( 24 weeks) of the experiment. Each group consisted of 10 rats. $* p<0.05$, $* * p<0.01, * * * p<0.001$ vs diabetic rats. No significant difference between diabetic and diabetic/troglitazone-high

cal scavenger was also shown in our results, i. e. the increased serum and nerve lipoperoxide in diabetic rats was inhibited by troglitazone. In addition, the enhanced TNF- $\alpha$ production in diabetic rats was also inhibited in the rats given both low and high doses of troglitazone. Recently, PPAR- $\gamma$ ligands, including troglitazone, have been reported to inhibit TNF- $\alpha$ production by macrophages activated in vitro with phorbol ester or okadaic acid but not LPS [22, 23]. These data are not incompatible with our observation that troglitazone did, but not strongly, inhibit the enhanced LPS-induced production of TNF- $\alpha$ under the chronic hyperglycaemic state. Since a sensitive assay of TNF- $\alpha$ such as ELISA was unavailable in rats, serum TNF- $\alpha$ was measured after LPS stimulation in our experiment. In vivo TNF- $\alpha$ production with or without stimulation by other than LPS might be more strongly inhibited by troglitazone.

Scavenging of free radicals and TNF- $\alpha$ inhibition by troglitazone could have ameliorated diabetic neuropathy possibly by suppressing the impairment of the microcirculation induced by free radicals $[20,21,24]$

or TNF- $\alpha$ [25] or both, although the relative contributions of these effects to the amelioration of neuropathy is obscure. These mechanisms could be involved in our findings showing that the improvement of MNCV by troglitazone was statistically significant after the week 12 or 16 of the experiment, although troglitazone was given from the beginning of the experiment. This implies that troglitazone treatment can affect the later phase of neuropathy, i.e. inhibiting exacerbation of the neuropathy rather than preventing initial events.

In conclusion, treatment with troglitazone inhibited the slowing of MNCV and morphological changes of the peripheral nerve in diabetic rats irrespective of blood glucose concentrations in STZ-induced diabetic rats.

Acknowledgements. This work was supported in part by a grant for Diabetes Research from the Ministry of Health and Welfare of Japan. We thank Dr. R. Wada and Professor S. Yagihashi, 1st Department of Pathology, Hirosaki University School of Medicine, for advice on morphometric analysis of the nerve, Professor T. Takahashi, Department of Pathology, Institute of Development, Ageing and Cancer, Tohoku University, for their generous help with the digital camera equipment and Mr. Y. Kumagai for his technical assistance. 


\section{References}

1. Cameron NE, Cotter MA, Basso M, Hohman TC (1997) Comparison of the effects of inhibitors of aldose reductase and sorbitol dehydrogenase on neurovascular function, nerve conduction and tissue polyol pathway metabolites in streptozotocin-diabetic rats. Diabetologia 40: 271-281

2. Gries AF (1995) Alternative therapeutic principles in the prevention of microvascular and neuropathic complications. Diabetes Res Clin Pract 28: 201-207

3. Tanaka S, Seino H, Satoh J et al. (1992) Increased in vivo production of tumour necrosis factor after development of diabetes in nontreated, long-term diabetic BB rats. Clin Immunol Immunopathol 62: 258-263

4. Sagara M, Satoh J, Zhu XP et al. (1994) Inhibition with Nacetylcysteine of enhanced production of tumour necrosis factor in streptozotocin-induced diabetic rats. Clin Immunol Immunopathol 71: 333-337

5. Nishimura S, Satoh J, Toyota T (1997) Serum TNF- $\alpha$ levels and diabetic complications in NIDDM patients. Diabetologia 40 [Suppl 1]:A601

6. Sagara M, Satoh J, Wada R et al. (1996) Inhibition of development of peripheral neuropathy in streptozotocin-induced diabetic rats with $\mathrm{N}$-acetylcysteine. Diabetologia 39: 263-269

7. Qiang X, Satoh J, Sagara M et al. (1995) Treatment of diabetic neuropathy with pentoxifylline. J Jpn Diabete Soc 38 [Suppl]:378

8. Lehmann JM, Moore LB, Smith-Oliver TA, Wilkison WO, Willson TM, Kliewer SA (1995) An antidiabetic thiazolidinedione is a high affinity ligand for peroxisome proliferator-activated receptor $\gamma(\operatorname{PPAR} \gamma)$. J Biol Chem 270: 12953-12956

9. Fujiwara T, Yoshioka S, Yoshioka T, Ushiyama I et al. (1988) Characterization of new oral antidiabetic agent troglitazone. Diabetes 37: 1549-1558

10. Iwamoto Y, Kuzuya T, Matsuda A et al. (1991) Effects of new oral hypoglycemic agent CS-045 on glucose tolerance and insulin secretion in patients with NIDDM. Diabetes Care 14: 1083-1086

11. Scott NA, Jennings PE, Brown J et al. (1991) Troglitazone: a general free radical scavenger. Eur J Pharmacol 208: 175-177

12. Jennings PE, Scott NA, Saniabadi AR et al. (1992) Effects of troglitazone on platelet reactivity and free radicals in type II diabetic patients: clinical assessment. Metabolism 41: 36-39

13. Noguchi N, Sakai H, Kato Y et al. (1996) Inhibition of oxidation of low density lipoprotein by troglitazone. Atherosclerosis 123: 227-234

14. Ohsumi J, Sakakibara S, Yamaguchi J et al. (1994) Troglitazone prevents the inhibitory effects of inflammatory cytokines on insulin-induced adipocyte differentiation in 3T3L1 cells. Endocrinology 135: 2279-2282

15. Szalkowski D, White-Carrington S, Berger J, Shang B (1995) Antidiabetic thiazolidinediones block the inhibitory effect of tumour necrosis factor- $\alpha$ on differentiation, insulin-stimulated glucose uptake, and gene expression in 3T3L1 cells. Endocrinology 136: 1474-1481

16. Yagihashi S, Kamijo M, Ido Y, Mirrlees DJ (1990) Effects of long-term aldose reductase inhibition on development of experimental diabetic neuropathy. Diabetes 39: 690-696

17. Yagi K (1976) A simple fluorometric assay for lipoperoxide in blood plasma. Biochemical Medicine 15: 212-216

18. Satoh J, Seino H, Abo T et al. (1989) Recombinant human tumour necrosis factor- $\alpha$ suppresses autoimmune diabetes in nonobese diabetic mice. J Clin Invest 84: 1345-1348

19. Sima AAF, Brown MB, Prashar A, Chakrabarti S, Laudadio C, Greene DA (1992) The reproducibility and sensitivity of sural nerve morphometry in the assessment of diabetic peripheral polyneuropathy. Diabetologia 35: 560-569

20. Cotter MA, Love A, Watt MJ, Cameron NE, Dines KC (1995) Effects of natural free radical scavengers on peripheral nerve and neurovascular function in diabetic rats. Diabetologia 38: 1285-1294

21. Low PA, Nickander KK, Tritschler HJ (1997) The roles of oxidative stress and antioxidant treatment in experimental diabetic neuropathy. Diabetes 46 [Suppl 2]:S38-S42

22. Ricote M, Li AC, Willson TM, Kelly CJ, Glass CK (1998) The peroxisome proliferator-activated receptor- $\gamma$ is a negative regulator of macrophage activation. Nature 391: 79-82

23. Jiang C, Ting AT, Seed B (1998) PPAR- $\gamma$ agonists inhibit production of monocyte inflammatory cytokines. Nature 391: 82-86

24. Langenstroer P, Pieper GM (1992) Regulation of spontaneous EDRF release in diabetic rat aorta by oxygen free radicals. Am J Physiol 263: H257-H265

25. Dawson DA, Martin D, Hallenbeck JM (1996) Inhibition of tumour necrosis factor-alpha reduces focal cerebral ischemic injury in the spontaneously hypertensive rat. Neurosci Lett 218: 41-44 